\title{
Infezione da HCV: quale differenza di approccio in politica sanitaria se l'obiettivo è la guarigione $\mathrm{HCV}$ infection: which approach for the policy maker if the goal is healing
}

\begin{abstract}
Lucrezia Ferrario ${ }^{1}$, Emanuela Foglia ${ }^{1}$, Elisabetta Garagiola ${ }^{1}$, Davide Croce $^{1,2}$
${ }^{1}$ Centro di Ricerca in Economia e Management in Sanità e nel Sociale, LIUC - Università Cattaneo, Castellanza, Italia; ${ }^{2}$ School of Public Health, Faculty of Health Sciences, University of the Witwatersrand, Johannesburg, South Africa.
\end{abstract}

\section{Abstract}

The Italian National Healthcare Service decision makers are currently facing economic and financial problems, in particular related to the budget impact of HCV infection and the correct planning of the limited available resources. Two are the main difficulties: the estimate of HCV population, and the evolution of budget for the treatments innovation.

The primary objective of the present contribution was the identification of the methodological approaches available to decision makers, within the context of HCV, outlining strengths and weaknesses. Three main methodological approaches were identified: i) health economics evaluations; ii) budget impact models and iii) the multidimensional models such as the Health Technology Assessment.

The cost-effectiveness analysis is not able to provide complete and unambiguous indications for a decision maker, in terms of short and long term sustainability of planning and control activities; the budget impact analysis is not useful as information for the patients. The two methods, however, are complementary to each other: the use of multidimensional and multidisciplinary approaches could guarantee all the information useful for the implementation of strategies that could heal the largest number of people.
Autore

per la corrispondenza:

Elisabetta Garagiola

Corso Matteotti, 22 21053 Castellanza (VA) Italy

T. 00390331572467

Fax 00390331572513 egaragiola@liuc.it

Keywords:

Health Economics Evaluation, Budget Impact Analysis - BIA, Health Technology Assessment HTA, HCV infection, decision making JHA 2016; 1(2): 26-32 DOI: 10.19198/JHA31407

\section{Introduzione: \\ Aspetti legati alla popolazione}

Il fenomeno dell'infezione dal virus dell'epatite C (HCV) sta assumendo una portata rilevante, tanto da essere stato riconosciuto nel 2010, da parte dell'Organizzazione Mondiale della Sanità, come uno dei principali problemi sanitari a impatto globale. 
La letteratura riporta che, a livello epidemiologico, circa il $2.35 \%$ della popolazione mondiale viene colpita da tale condizione morbosa grave (1) con conseguenze cliniche acute (quali cirrosi epatiche, epatocarcinomi, trapianti di fegato etc.) e croniche (2).

Tale dato di prevalenza, però, presenta una certa variabilità a livello complessivo: non è certo quanto esso si discosti nei differenti contesti locali (1). Tale incertezza è dovuta in primis alle difficoltà di diagnosticare l'infezione da HCV, con una significativa possibilità di sottostimare gli esordi della patologia: le fasi meno avanzate sono, infatti, clinicamente silenti. Molto spesso è necessario attendere anche più di trent'anni per la progressione della patologia: è noto come molti pazienti infettatisi negli anni '90 siano ancora attualmente asintomatici (3). Si aggiunga a ciò anche il fatto che le recrudescenze e la progressione dell'infezione verso stadi più avanzati sono poco prevedibili e dipendenti da fattori ambientali, legati alla sfera del paziente, quali età, sesso, comorbidità, coinfezione con HIV o HBV (4).

L'Italia e la Romania sono in Europa i paesi a maggiore prevalenza, con circa 1,8 milioni di soggetti HCV positivi (5) e l'Italia è quello con il tasso di mortalità più elevato per epatocarcinoma e cirrosi (2).

Non esistono però delle evidenze nazionali univoche: in letteratura sono presenti alcuni studi (6-8) che forniscono informazioni in merito alla prevalenza a livello di aree geografiche italiane $(7.3 \%$ nelle regioni del sud, $6.1 \%$ in quelle del centro, fino all'1.6\% del nord Italia), o di differenti fasce di popolazione (si hanno risultati differenti se si considerano, per esempio, le coorti di pazienti nate prima degli anni '50 o '60 dello scorso secolo, o nella popolazione giovanile), ma un quadro definitivo ed esaustivo non è a oggi disponibile (5).

Risultato ultimo è una continua sottostima dei pazienti che possono intraprendere dei percorsi di trattamento innovativo, oppure ancora l'emersione di un sommerso di pazienti che, soprattutto con i nuovi trattamenti, negli ultimi anni, tendono a venire allo scoperto e a incrementare il numero di nuove diagnosi.

È facilmente intuibile come le difficoltà di stima della popolazione affetta da HCV abbiano delle ripercussioni sui percorsi decisionali del management sanitario, soprattutto in riferimento alla fase di pianificazione e programmazione dell'attività sanitaria, così da non agevolare lo svolgersi di azioni volte a un utilizzo mirato e ottimale delle risorse sanitarie.

Allo stato attuale, le sole evidenze scientifiche di stampo sia clinico, sia economico sanitario, generate negli ultimi anni $(9,10)$, forniscono un quadro di patologia aggregato di una popolazione che è assai eterogenea e che presenta quindi problematiche e un assorbimento di risorse completamente differente.

\section{Popolazione e tecnologie: elementi di base per il policy making}

I decision maker devono tenere in considerazione il processo di evoluzione dell'innovazione tecnologica. Le terapie farmacologiche anti-HCV si sono da sempre poste come obiettivo ultimo la capacità di raggiungere una risposta virologica sostenuta (Sustained Virologic Response - SVR) in tempistiche il più contenute possibili.

A partire dalla metà dell'anno 2015, sono state introdotte sul mercato nuove alternative terapeutiche (Direct Acting Antiviral-DAA ), con caratteristiche innovative quali la possibilità di poter somministrare il farmaco con schemi terapeutici più semplici o addirittura in co-formulazione dei principi attivi, attraverso un'unica compressa per una mono-somministrazione giornaliera, ma elemento più importante tra tutti, l'opportunità di una cura senza iniezione sottocute di Interferone Pegilato (PEG-Inf) e con durate di trattamento inferiori. Le strategie terapeutiche tradizionali alla lotta all'epatite $C$, invece, si sono sempre presentate con degli schemi di assunzione molto complessi, tanto da avere il potere di condizionare notevolmente il livello di aderenza del paziente e il raggiungimento della SVR. È noto in letteratura, infatti, come la diminuzione del numero di pillole possa essere associata a una maggiore aderenza da parte del paziente, e, quindi a una maggiore efficacia del trattamento (12).

Ponendo, poi, l'attenzione sul tema delle risorse e, pertanto, sui fattori più specificatamente economici, va sottolineato come tali trattamenti innovativi siano a elevato costo e non rappresenterebbero I'unico costo a carico del Servizio Sanitario Nazionale (SSN) per l'HCV. Nella logica di comprendere i costi correlati alla cura e al trattamento del paziente, sono da considerare anche tutte quelle 
prestazioni connesse alla diagnosi, presa in carico e cura della patologia medesima, soprattutto in riferimento alle sequele patologiche HCV-correlate $(12,13)$.

Aggrava e complica il quadro il fatto che il SSN italiano, di tipo universalistico, sta attraversando da alcuni anni una fase di continue azioni di spending review e tagli lineari, a causa della recente crisi economica che ha investito in senso generale il nostro sistema paese (con un PIL praticamente immobile da diverso tempo), il mondo della Pubblica Amministrazione, includendo il settore sanitario in modo particolare.

Pertanto dall'indeterminatezza nella stima della popolazione HCV e dall'evoluzione della patologia dovuta all'innovazione, ne consegue un problema economico e finanziario, di pianificazione delle necessità legate all'infezione da HCV in sede di budget, regionale o nazionale, oppure ancora a livello di singola azienda ospedaliera o sanitaria erogante le correlate prestazioni di trattamento e cura, al fine di una sostenibilità globale deI SSN italiano.

\section{Obiettivo del contributo}

Come i decision maker dovrebbero approcciare, dunque, questa ampia tematica nell'impossibilità di autogenerare le informazioni in merito alla stima della popolazione affetta da HCV e nella difficoltà di trattare tutti i pazienti con farmaci innovativi, a causa della stringente regolamentazione di sistema e della congiunturale situazione di scarsità e limitatezza di risorse? Quali approcci possono essere messi in campo, sotto un profilo economico-sanitario, di pianificazione e programmazione, al fine di prendere delle decisioni evidence-based e consistenti? E soprattutto... quale strumento potrebbe garantire un miglior raggiungimento delle istanze di sistema, ponendo, come obiettivo ultimo e preponderante, il fornire risposte adeguate alle esigenze del paziente e garantire la sua guarigione?

A fronte del contesto delineato in precedenza, e dei gap conoscitivi, sotto un profilo epidemiologico e di policy making, il presente contributo intende raggiungere il seguente obiettivo: effettuare una disamina degli approcci metodologici a disposizione dei decisori di spesa, che debbono affrontare importanti processi di programmazione e presa di decisioni all'interno del contesto dell'HCV, delineando gli elementi di forza e di debolezza, avendo come finalità ultima la migliore gestione del paziente e la sua guarigione.

\section{Identificazione degli approcci metodologici a supporto dei decision maker} All'interno dello specifico contesto dell'HCV, si sono identificati fondamentalmente tre principali approcci metodologici di potenziale interesse: i) la sfera delle valutazioni economico-sanitarie, intese come applicazione di analisi comparative

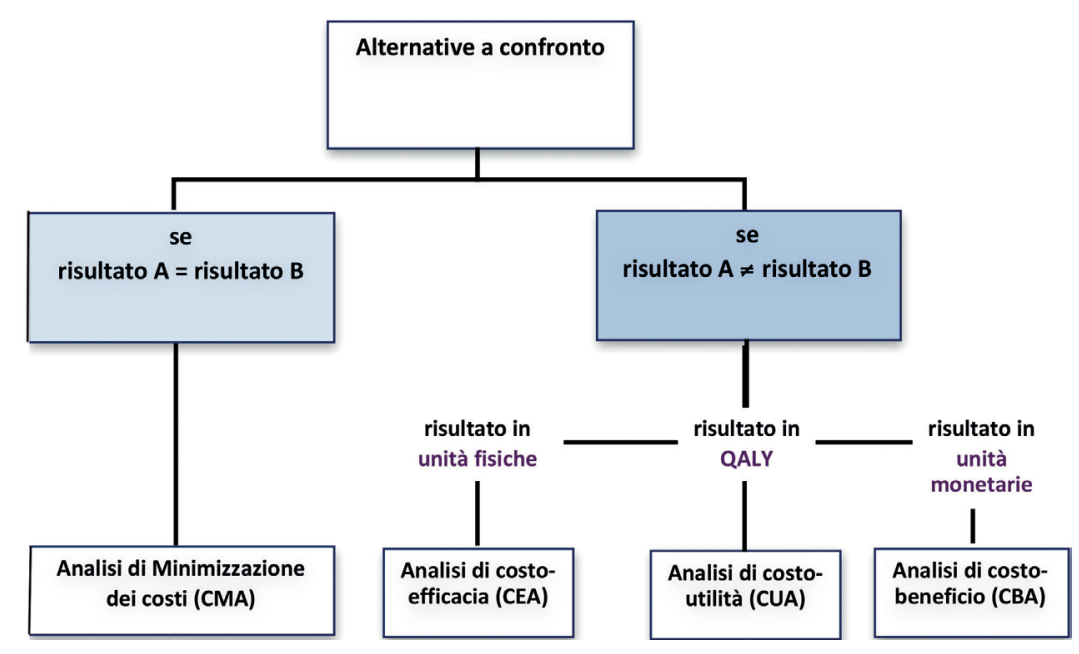

Figura 1. Metodiche di Valutazione Economica a confronto 
delle alternative tecnologiche in termini di costi e di conseguenze degli interventi sanitari (14); ii) i modelli di budget impact intesi come la stima delle conseguenze finanziarie dell'adozione e della diffusione di nuove tecnologie sanitarie all'interno di uno specifico setting o sistema $(15,16)$; iii) infine i modelli multidimensionali quali l'Health Technology Assessment, ovvero strumenti multidisciplinari in grado di studiare le implicazioni cliniche, sociali, etiche ed economiche dello sviluppo, diffusione e utilizzo di una tecnologia sanitaria (17).

Il primo approccio, ovvero quello delle Valutazioni Economico-Sanitarie (VES), è in grado di coniugare e correlare, all'interno dei processi sanitari, l'utilizzo di risorse e gli output prodotti, con gli outcome generati dal consumo di quelle specifiche risorse, garantendo una specifica prestazione, in modo tale da fornire un supporto alla razionale allocazione delle risorse. Caratteristica di una VES è quella di confrontare almeno due alternative differenti, e procedere con una modalità di approccio comparativa (18). Sotto il cappello delle VES vengono incluse cinque tecniche da utilizzare diversamente a seconda della misura di efficacia dei comparator (Figura 1) Se le alternative tecnologiche che si stanno studiando presentano la medesima efficacia, significa che è possibile garantire la stessa risposta in termini di salute, a prescindere dall'alternativa scelta: in questi casi si utilizza come unico criterio di discernimento quello economico, preferendo, a parità di efficacia, la alternativa con il minore costo (conducendo quindi una Analisi di Minimizzazione dei costi - CMA). Se invece i comparator analizzati presentano una efficacia differenziale, sarà necessario prendere in considerazione non solo le risorse utilizzate, quindi i costi, ma anche le conseguenze degli interventi sanitari (Analisi di costo-efficacia (CEA) (19), Analisi di costo-utilità (CUA), Analisi di costo-beneficio (CBA) (20)).

Il secondo approccio a supporto dei decision maker è rappresentato dai modelli di budget impact: questa tecnica permette di predire quale impatto potrà avere sulla spesa l'introduzione di una nuova tecnologia sanitaria o una modifica nel mix di utilizzo di tecnologie sanitarie sia a livello regolatorio (nazionale o regionale), per la gestione e pianificazione dei budget sanitari, sia a livello locale, per valutare la sostenibilità di investimenti tecnologici $(15,16)$. Tale strumento compara, in termini di costi diretti sanitari, lo scenario attuale e quello innova- tivo, tipicamente su un orizzonte temporale di breve-medio periodo, 3 o 5 anni (poiché la prospettiva è quella del budget holder, che non presenta mai un orizzonte di azione e di mandato più ampio).

Un'analisi di impatto sul budget (Budget Impact Analysis BIA) prevede una attenta disamina e valutazione degli estremi, normalmente definiti come proiezione di minimo o di massimo, oppure ancora categorizzati come best case e worst case, per comprendere all'interno di quale range si collocherà il risultato finale.

Tale strumento può fornire all'intero sistema importanti indicazioni, andando a strutturare e costruire delle analisi di scenario, modificando le variabili e le determinanti all'interno del mix di trattamenti presi in considerazione, oppure le condizioni di popolazione trattata, o sulla scorta della volontà di testare differenti ipotesi che potrebbero ugualmente occorrere, al fine di fornire un potenziale quadro di chiarezza, a fronte di una incertezza degli elementi di base che vanno a popolare il modello $(15,16)$, esattamente come può avvenire nel caso dell'HCV.

Il terzo approccio metodologico a cui i decision maker possono fare riferimento è quello dei modelli multidimensionali, quali l'Health Technology Assessment (HTA), che permettono di valutare, sotto diverse prospettive, l'impiego delle tecnologie in ambito sanitario (21), avendo sempre a riferimento almeno un'alternativa esistente.

È proprio l'approccio multidimensionale e multidisciplinare la caratteristica peculiare della metodica dell'HTA, il cui obiettivo primario è quello di salvaguardare e migliorare la salute della popolazione nonché l'appropriatezza nell'utilizzo di tecnologie sanitarie, analizzando, con metodo scientifico, le implicazioni cliniche, sociali, etiche, organizzative ed economiche dello sviluppo, introduzione e diffusione di una determinata tecnologia o intervento sanitario, definendo così strategie che aiutino l'implementazione delle politiche sanitarie (22). Se infatti l'applicazione di valutazioni economico-sanitarie prende in considerazione due sole dimensioni (economica e di efficacia) e la BIA addirittura esclusivamente quella economico-finanziaria (pur fornendo una importante informazione di popolazione e di scenario), I'HTA esprime la capacità di approcciare al tema della valutazione della tecnologia, prendendo in considerazione, nella peggiore delle ipotesi, quattro dimensioni di osservazione, 
quella economica, di efficacia, del paziente e organizzativa (23), nei modelli di HTA più semplificati. Ulteriore stimolo all'utilizzo di questo strumento deriva dal fatto che l'approccio dell'Health Technology Assessment è ormai consolidato nelle prassi regionali italiane, ma soprattutto è investito di un livello di istituzionalizzazione anche nazionale, che le altre metodiche non hanno. Non da ultimo, fattore di assoluto rilievo è legato al fatto che I'HTA ingloba, nella sua applicazione, all'interno della dimensione economica, l'effettuazione sia di una valutazione economico-sanitaria completa, sia la conduzione di un'analisi di budget impact. II Patto di Stabilità 2016 affronta pesantemente questo argomento, andando a suggerire l'utilizzo di un sistema di HTA che sia assimilabile a quello europeo, attraverso un modello a 9 dimensioni di indagine chiamato Modello EUnetHTA (2015), che ha la finalità di analizzare: i) la rilevanza della tecnologia, ii) la rilevanza della patologia, iii) la sicurezza della tecnologia oggetto di indagine e del comparator o dei comparator di riferimento, iv) l'effectiveness e/o l'efficacy, v) l'impatto economico finanziario (comprendendo sia la conduzione di una VES sia della BIA), vi) impatto sociale ed etico (assumendo il punto di vista del paziente), vii) impatto di equità, viii) impatto organizzativo qualitativo e quantitativo (ossia che vada a indagare sia la percezione degli utenti interni, sia anche che quantifichi l'eventuale necessità di investimenti specificatamente correlati a formazione, adeguamento strutturale o altro elemento che possa impattare sul budget), ix) impatto legale (24).

\section{Conclusioni}

In questa sede pare opportuno riflettere sui punti di forza e di debolezza degli approcci metodologici identificati, alla luce soprattutto della loro sperimentazione, nel contesto dell'infezione da HCV.

Le VES, nello specifico l'analisi di costo-efficacia, e la BIA hanno caratteristiche diverse, come evidenziato e sintetizzato nella Tabella 1, giacché partono da presupposti differenti. Una valutazione economico-sanitaria tende a fornire una visione statica, di un quadro di popolazione che normalmente è dinamico, andando a considerare normalmente un dato medio a paziente. Non è quindi in grado di fornire un'indicazione esaustiva e univoca per un decision maker, in merito alla sostenibilità dell'implementazione della tecnologia sanitaria oggetto

\begin{tabular}{|c|c|c|}
\hline & $\begin{array}{l}\text { Analisi di impatto } \\
\text { sul budget }\end{array}$ & $\begin{array}{c}\text { Analisi di costo } \\
\text { effiacia }\end{array}$ \\
\hline Prospettiva dello studio & Budget holder & $\begin{array}{l}\text { Variabile (società, servizio } \\
\text { sanitario, paziente) }\end{array}$ \\
\hline Alternative & Attuale mix di trattamenti & Una o più tecnologie \\
\hline Orizzonte temporale & $\begin{array}{l}\text { Breve - medio termine } \\
\text { (1-5 anni) }\end{array}$ & Variabile (breve, medio, lungo) \\
\hline Discounting & Non necessario & $\begin{array}{l}\text { Da applicare a studi } \\
\text { con orizzonte } \\
\text { temporale medio/lungo }\end{array}$ \\
\hline Costi & Costi sanitari diretti & $\begin{array}{l}\text { Costi diretti (sanitari e non } \\
\text { sanitari) e costi indiretti, in base } \\
\text { alla prospettiva assunta }\end{array}$ \\
\hline Efficacia & $\begin{array}{l}\text { Costi e risparmi } \\
\text { di breve termine }\end{array}$ & $\begin{array}{l}\text { Unità fisiche naturali } \\
\text { e qualità della vita }\end{array}$ \\
\hline Risultati & Costi sanitari incrementali & $\begin{array}{l}\text { Rapporto di costo-efficacia } \\
\text { incrementale }\end{array}$ \\
\hline Analisi di sensibilità & $\begin{array}{l}\text { Analisi degli estremi } \\
\text { e analisi di scenario }\end{array}$ & $\begin{array}{c}\text { Differenti tipologie } \\
\text { di analisi (univariata, } \\
\text { multivariata, analisi di scenario, } \\
\text { probabilistica, etc...) }\end{array}$ \\
\hline
\end{tabular}

Tabella 1. Confronto tra analisi di impatto sul budget e analisi di costo-efficacia. Fonte: adattata da Garattini et al. (25).

di indagine, poiché restituisce solamente un dato medio di confronto diretto tra alternative, non riuscendo da sola a considerare il mix di alternative a disposizione. A fronte di ciò, per essere veramente utilizzata all'interno del processo decisionale, necessita di essere accompagnata da un'analisi complementare, come può essere quella di impatto sul budget.

La BIA è in grado di affrontare la valutazione tecnologica secondo un orizzonte temporale coerente rispetto al processo di presa di decisioni, cogliendo certe dinamiche che un'analisi di costo-efficacia non fornisce sempre puntualmente: in alcuni casi ciò avviene perché la VES fruisce di dati reperiti di breve periodo (anche solo semestrali), mentre può accadere anche che una valutazione economico-sanitaria sia proiettata in un orizzonte temporale life-time, ossia che prenda in considerazione tutta la vita del paziente, con notevoli problematiche di comprensione dei benefici generati in orizzonti temporali-decisionali accettabili e standard, come possono essere i 12-24-36 mesi.

Le due metodiche possono però essere assolutamente complementari tra loro. Si potrebbe agire nella logica di comprendere in un primo momento, 
attraverso la VES, quali alternative dovrebbero essere a maggiore valore aggiunto, in modo tale da inserire le medesime all'interno di una più ampia analisi di budget impact per indagare l'impatto complessivo finale del cambio di mix così delineato.

Ulteriore possibilità potrebbe essere quella di valutare il migliore indicatore di costo-efficacia, rispetto alla stratificazione della popolazione, e andare a comprendere i benefici economici correlati al trattamento di una maggiore fetta di popolazione a maggiore vantaggio per il sistema. Purtroppo però, se il primo approccio può essere facilmente e tecnicamente implementabile, nonché comunicabile senza problematiche di sistema, il secondo approccio nasconde maggiori insidie, per una paura di generare iniquità all'interno del sistema, anche se sarebbe l'approccio in grado di generare maggiore vantaggio per i pazienti, ma soprattutto per la società complessivamente.

La BIA di per sé non fornisce un dato agevolmente leggibile da parte del paziente, ma al tempo stesso è una preziosa fonte di informazioni per i decision maker, in merito all'entità dell'investimento che il Sistema Sanitario Regionale dovrebbe intraprendere per il trattamento dei pazienti HCV e alle relative questioni di liquidità nel breve periodo. All'interno di un modello di budget impact come quello proposto, se si provasse ad ampliare il campione dei pazienti trattati con delle analisi di scenario sia regionali sia soprattutto nazionali, i decision maker potrebbero effettivamente valutare quali soggetti eleggere al trattamento, dopo aver compreso le ricadute economiche: è noto, infatti, come, all'aumentare della popolazione trattata, si potrebbero ottenere delle importanti scontistiche di prezzo dei trattamenti a fronte di volumi più ingenti, uti- lizzando favorevolmente per il sistema gli accordi prezzo-volume in essere tra sistema e produttori, centralizzando quindi le scelte a vantaggio di tutti i pazienti.

È solo quindi, con l'unione di più approcci metodologici che si ha la possibilità di meglio governare i dati e le proiezioni del sistema, con la finalità ultima di poter garantire l'applicazione di quelle strategie che possano guarire il numero più grande di persone possibile e al tempo stesso porre una pietra miliare per l'eradicazione del virus.

Ulteriori elementi potrebbero essere ancora integrati agli approcci metodologici delle VES e dalla BIA per fornire un quadro completo della tecnologia sanitaria da implementare: in questo caso si vuole fare riferimento alla possibilità di implementazione di una valutazione di HTA completa, che prenda in considerazione tutte le dimensioni richieste dal Patto di Stabilità.

Con questo approccio non solo si fornirebbero tutte le informazioni di sistema ai decision maker, ma si potrebbe avere l'opportunità di rilevare anche il punto di vista del paziente, comprendendo il reale vantaggio percepito dallo stesso e rendendolo protagonista attivo del processo di valutazione e non solamente protagonista "passivo", attraverso i suoi dati e le informazioni rilevate dai flussi. Questo potrebbe consentire di valutare la dimensione della qualità di vita percepita o degli aspetti soggettivi che comunque devono essere elementi da comprendere al fine di creare commitment all'interno del sistema e una comunicazione maggiormente efficace, elementi che, con la medesima dignità degli argomenti economici, regolatori e di efficacia, generano sicurezza e migliorano il percorso di presa in carico del paziente, rendendolo più lineare e, quindi, più appropriato.

\section{BIBLIOGRAFIA}

1. Lavanchy D. The global burden of hepatitis C. Liver Int 2009; 29 (Suppl 1): 74-81.

2. European Association of the Study of the Liver. European Association of the Study of the Liver hepatitis C virus clinical practice guidelines, Liver Int 2012; 32: 2-8.

3. Davis GL, Alter MJ, El-Serag H, et al. Aging of hepatitis C virus (HCV)-infected persons in the United States: a multiple cohort model of HCV prevalence and disease progression. Gastroenterol 2010; 138: 513-21.

4. Zaltron S, Spinetti A, Biasi L, et al., Chronic HCV infection: epidemiological and clinical relevance. BMC Infect Dis 2012; 12 (Suppl 2): S2. 


\section{appropriateness paper}

5. AISF-FIRE. Libro bianco dell'epatologia italiana, 2011. (www.webaisf.org/media/13891/libro-bianco-aisf-2011.pdf)

6. Mariano A, Scalia Tomba G, et al. Estimating the incidence, prevalence and clinical burden of hepatitis C over time in Italy. Scand J Infect Dis 2009; 41: 689-99.

7. Ansaldi F, Bruzzone B, Salmaso S, et al. Different seroprevalence and molecular epidemiology patterns of hepatitis $C$ virus infection in Italy. J Med Virol 2005; 76: 327-32.

8. Alberti A, Noventa F, Benvegnù L, et al. Prevalence of liver disease in a population of asymptomatic persons with hepatitis $C$ virus infection. Ann Intern Med 2002; 137: 961-4.

9. Mennini FS, Marcellusi A, Andreoni M, et al. Health policy model: long-term predictive results associated with the management of hepatitis C virus-induced diseases in Italy. ClinicoEcon Outcomes Res 2014; 6: 303-10.

10. Razavi H, Elkhoury AC, Elbasha E, et al. Chronic hepatitis C virus (HCV) disease burden and cost in the United States. Hepatology, 2013; 57: 2164-70.

11. Murri R, Fantoni M, Del Borgo C, et al. Determinants of health-related quality of life in HIV-infected patients. AIDS Care 2003; 15: 581-90.

12. Alter HJ, Seeff LB. Recovery, persistence, and sequelae in hepatitis C virus infection: a perspective on long-term outcome. Semin Liver Dis 2000; 20: 17-35.

13. Lauer GM, Walker BD. Hepatitis C virus infection. N Engl J Med 2001; 345: 41-52.

14. Drummond MF, Schwartz JS, Jönsson B, et al. Key principles for the improved conduct of health technology assessments for resource allocation decisions. Int J Technol Assess Health Care, 2008; 24: 244-58.

15. Mauskopf JA, Sullivan SD, Annemans L, et al. Principles of good practice for budget impact analysis: report of the ISPOR Task Force on good research practices--budget impact analysis. Value Health. 2007; 10: 336-47.

16. Sullivan SD, Mauskopf JA, Augustovski F, et al. Budget impact analysis-principles of good practice: report of the ISPOR 2012 Budget Impact Analysis Good Practice II Task Force. Value Health 2014; 17: 5-14.

17. International Network Of Agencies For Health Technology Assessment (INAHTA). Health Technology Assessment (HTA) Glossary. Stockholm; 2006. (www.sbu.se/filer/content11/ dokument/Edu_INHTA_glossary_2006_final).

18. Associazione Italiana di Economia Sanitaria (AIES). Proposta di linee guida per la valutazione economica degli interventi sanitari. PharmacoEconomics - Italian Research Articles, 2009; 11: 83-93.

19. Detsky AS, Naglie IG. A Clinician's Guide to Cost-effectiveness Analysis. Ann Int Med 1990; 113: 147-54.

20. Johannesson $\mathrm{M}$, Aberg $\mathrm{H}$, Agreus $\mathrm{H}$, et al. Cost-benefit analysis of non-pharmacological treatment of hypertension. J Int Med 1991; 230: 307-12.

21. Sacchini D, Refolo P. L'Health Tecnology Assessment e i suoi aspetti etici. Medicina e Morale, 2007/I; 101-139.

22. Ricciardi W, Cicchetti A, Marchetti M. Health Technology Assessment's Italian Network: Origins, aims and advancement. It J Public Health, 2005; 2: 29-33. (www.ijphjournal.it/ article/view/5981/5726)

23. Kidholm K, Ehlers L, Korsbek L, et al. Assessment of the quality of mini-HTA. Int J Technol Assess Health Care 2009; 25: 42-48.

24. EUNetHTA, The HTA Core Model, Version 2.1, Aprile 2015. (www.meka.thl.fi/htacore/model/HTACoreModel2.1.pdf).

25. Garattini L, van de Vooren K. Budget impact analysis in economic evaluation: a proposal for a clearer definition. Eur J Health Econ. 2011; 12:499-502. doi: 10.1007/s10198-011-0348-5. 